\title{
REFERENCES
}

1. L. V. Ahlfors and Leo Sario, Riemann surfaces, Princeton Univ. Press, Princeton, N. J., 1960.

2. H. B. Curtis, Jr., Some properties of functions which uniformize a class of simply connected Riemann surfaces, Proc. Amer. Math. Soc. 10 (1959), 525-530.

3. - The uniformization of a class of simply connected Riemann surfaces, Proc. Amer. Math. Soc. 11 (1960), 511-516.

4. G. R. MacLane, Riemann surfaces and asymptotic values associated with real entire functions, The Rice Institute Pamphlet, Special Issue, November, 1952.

5. Rolf Nevanlinna, Eindeutige analytische Funktionen, Springer, Berlin, 1936.

6. H. E. Taylor, Determination of the type and properties of the mapping function of a class of doubly-connected Riemann surfaces, Proc. Amer. Math. Soc. 4 (1953), 52-68.

UNIVERSITY OF TEXAS AND

Texas Technological College

\section{THE ADJOINT OF A DIFFERENTIAL OPERATOR WITH INTEGRAL BOUNDARY CONDITIONS}

ALLAN M. KRALL

In [1] a second-order differential operator was defined on those functions in $L^{2}(0, \infty)$ satisfying an integral-point type of boundary condition. An analysis of its spectrum and two "eigenfunction" expansions follows. Left unanswered was the problem of finding the adjoint operator and explaining where the nonhomogeneous expansion came from. We now derive the adjoint operator, classify its spectrum and show that the nonhomogeneous expansion is, in fact, the eigenfunction expansion associated with the adjoint operator. It is interesting to see that the adjoint operator is a combination of a differential operator and a one-dimensional vector in $L^{2}(0, \infty)$.

1. The operator $L$. We consider a differential expression of the form $l y=-y^{\prime \prime}+q(x) y, 0 \leqq x<\infty$, where $q(x)$ is an arbitrary measurable complex function satisfying $\int_{0}^{\infty}|q(x)| d x<\infty$.

We denote by $D_{0}$ those functions $f$ defined on $[0, \infty)$ and satisfying

1. $f$ is in $L^{2}(0, \infty)$,

2. $f^{\prime}$ exists and is absolutely continuous on every finite subinterval of $[0, \infty)$,

3. If is in $L^{2}(0, \infty)$.

Let $K(x)$ be an arbitrary complex-valued function on $L^{2}(0, \infty)$,

Received by the editors April 15, 1964. 
and let $\alpha$ and $\beta$ be complex numbers with $|\alpha|^{2}+|\beta|^{2} \neq 0$. We denote by $D$ those functions $f$ satisfying

1. $f$ is in $D_{0}$,

2. $\int_{0}^{\infty} K(x) f(x) d x-\beta f(0)+\alpha f^{\prime}(0)=0$.

We define the operator $L$ by $L f=l f$ for all $f$ in $D$. It was shown in [1] that $D$ is dense in $L^{2}(0, \infty)$ and that $L$ is a closed operator.

It was further shown that there exist solutions $y_{1}(x, s)$ and $y_{2}(x, s)$ of $(l-\lambda) y=0$, where $s=\lambda^{1 / 2}$ such that $0 \leqq \arg s \leqq \pi$ with the properties

$$
\begin{array}{ll}
y_{1}(x, s)=e^{i s x}(1+o(1)), & y_{1}^{\prime}(x, s)=e^{i s x}(i s+o(1)), \\
y_{2}(x, s)=e^{-i s x}(1+o(1)), & y_{2}^{\prime}(x, s)=e^{-i s x}(-i s+o(1))
\end{array}
$$

as $x \rightarrow \infty$ for all $s \neq 0$.

The eigenvalues of $L$ are given by $\lambda=s^{2}$ when $s$ is a zero in the upper half $s$-plane of

$$
N(s)=\int_{0}^{\infty} K(x) y_{1}(x, s) d x-\beta y_{1}(o, s)+\alpha y_{1}^{\prime}(o, s) .
$$

If $\int_{0}^{\infty}|K(x)| d x<\infty$ and $\alpha \neq 0$, the eigenvalues are bounded, at most countable in number, and accumulate only on the positive semi-axis $\lambda \geqq 0$. If $N(s)$ is never zero for all reals, then the eigenvalues are finite in number.

It was shown that except for the positive semi-axis $\lambda \geqq 0$ the remainder of the $\lambda$ plane determines the resolvent set of $L$ and that the positive semi-axis $\lambda \geqq 0$ constitutes continuous spectrum unless both $N(s)$ and $N(-s)$ vanish simultaneously. In that event it is possible for $L$ to have residual spectrum on the positive semi-axis $\lambda \geqq 0$.

Let

$$
\begin{aligned}
u(x, s)= & -\frac{1}{2 i s}\left[y_{2}(x, s) \int_{x}^{\infty} K(z) y_{1}(z, s) d z\right. \\
& \left.+y_{1}(x, s) \int_{0}^{x} K(z) y_{2}(z, s) d z+\left(\alpha y_{2}^{\prime}(0, s)-\beta y_{2}(0, s)\right) y_{1}(x, s)\right] .
\end{aligned}
$$

Let

$$
V_{1}(\lambda, x, \xi)=-u(\xi, s) y_{1}(x, s) / N(s)
$$

and let

$$
V_{2}(\lambda, x, \xi)= \begin{cases}-\frac{1}{2 i s} y_{1}(\xi, s) y_{2}(x, s) & \text { for } \xi>x \\ -\frac{1}{2 i s} y_{2}(\xi, s) y_{1}(x, s) & \text { for } \xi<x .\end{cases}
$$


Then the Green's function for $L$ is given by

$$
V(\lambda, x, \xi)=V_{1}(\lambda, x, \xi)+V_{2}(\lambda, x, \xi) .
$$

The resolvent for $L$ is the bounded integral operator with $V(\lambda, x, \xi)$ as its Kernel.

2. The adjoint operator $L^{*}$. We resort to a series of lemmas to find $L^{*}$.

Lemma 2.1. Iff is in $D_{0}$, then limit ${ }_{x \rightarrow \infty} f(x)=0$ and $\operatorname{limit}_{x \rightarrow \infty} f^{\prime}(x)=0$.

See [1, Lemma 4.1].

Lemma 2.2. If $g$ is in the domain of $L^{*}$ then $\bar{g}$ is in $D_{0}, \bar{g}(\xi)$ $=\int_{0}^{\infty} V(\lambda, x, \xi)\left(\left[\left(L^{*}-\bar{\lambda}\right) g(x)\right]\right)-d x$.

If $\lambda$ is not in the spectrum of $L$, we have

$$
f(x)=\int_{0}^{\infty} V(\lambda, x, \xi)(L-\lambda) f(\xi) d \xi \text { for all } f \text { in } D .
$$

If $g$ is in the domain of $L^{*}$, then

$$
\begin{aligned}
\int_{0}^{\infty}[(L-\lambda) f(\xi)] \bar{g}(\xi) d \xi=([L-\lambda] f, g)=\left(f,\left[L^{*}-\bar{\lambda}\right] g\right) \\
\quad=\int_{0}^{\infty} \int_{0}^{\infty} V(\lambda, x, \xi)[(L-\lambda) f(\xi)]\left(\left[\left(L^{*}-\bar{\lambda}\right) g(x)\right]\right)-d \xi d \dot{x} \\
=\int_{0}^{\infty}[(L-\lambda) f(\xi)]\left(\int_{0}^{\infty} \bar{V}(\lambda, x, \xi)\left(\left[\left(L^{*}-\bar{\lambda}\right) g(x)\right]\right)-d x\right) d \xi .
\end{aligned}
$$

Since $(L-\lambda) f$ may be arbitrary in $L^{2}(0, \infty)$ the integral follows. The differentiation is easily verified.

Lemma 2.3. If $g$ is in the domain of $L^{*}$, then

$$
\begin{gathered}
\left(L^{*} g(\xi)\right)^{-}=l \bar{g}(\xi)+\left(\frac{1}{N(s)}\right)\left(\int_{0}^{\infty}\left(\left[L^{*}-\bar{\lambda}\right] g(x)-y_{1}(x, s) d x\right) K(\xi),\right. \\
(g(0))^{-}=-\left(\frac{1}{N(s)}\right)\left(\int_{0}^{\infty}\left[\left(L^{*}-\bar{\lambda}\right] g(x)\right)-y_{1}(x, s) d x\right) \alpha, \\
\left(g^{\prime}(0)\right)^{-}=-\left(\frac{1}{N(s)}\right)\left(\int_{0}^{\infty}\left[\left(L^{*}-\bar{\lambda}\right] g(x)-y_{1}(x, s) d x\right) \beta .\right.
\end{gathered}
$$

These statements are easily verified by differentiation of the formula in Lemma 2.2. By eliminating the expressions in parentheses we are led to 
Theorem 2.4. If $g$ is in the domain of $L^{*}$, then

$$
L^{*} g=\bar{l} g-\mu_{0} \bar{K}, \quad \bar{\beta} g(0)-\bar{\alpha} g^{\prime}(0)=0,
$$

where $\mu_{o}=g(0) / \bar{\alpha}$ or $\mu_{o}=g^{\prime}(0) / \bar{\beta}$ or both depending on which is defined.

We denote by $E$ those functions $f$ defined on $(0, \infty)$ and satisfying

1. $f$ is in $D_{0}$,

2. $\beta f(0)-\alpha f^{\prime}(0)=0$,

3. If is in $L^{2}(0, \infty)$.

We define the operator $M$ by $M g=\bar{l} g-\mu_{g} \bar{K}$ for all $g$ in $\bar{E}$. We have shown so far that $L^{*} \subseteq M$.

THEOREM 2.5. $L^{*}=M$.

If $f \in D$ and $g \in \bar{E}$, then

$$
\begin{aligned}
(f, M g)= & \int_{0}^{\infty} f(x)\left[l(g(x))--\bar{\mu}_{\theta} K(x)\right] d x \\
= & {\left[f^{\prime}(x)(g(x))-f(x)\left(g^{\prime}(x)\right)-\right]_{0}^{\infty}+\int_{0}^{\infty}[l f(x)](g(x))-d x } \\
& -\bar{\mu}_{o} \int_{0}^{\infty} K(x) f(x) d x .
\end{aligned}
$$

Since the expression in the brackets vanishes at $\infty$ by Lemma 2.1, if $\alpha \neq 0$

$$
\begin{aligned}
(f, M g)= & \int_{0}^{\infty}[l f(x)](g(x))-d x \\
& -\frac{g(0)}{\alpha}\left[\int_{0}^{\infty} K(x) f(x) d x-\beta f(0)+\alpha f^{\prime}(0)\right] \\
= & (L f, g) \text { with a similar result if } \alpha=0 .
\end{aligned}
$$

Thus $M \subseteq L^{*}$ and $L^{*} g=\bar{l} g-\mu_{g} \bar{K}$ for all $g$ in $\bar{E}$.

3. The spectrum of $L^{*}$. The spectrum of $L$ is known. The problem, then, is to determine the spectrum of $L^{*}$ from that of $L$.

TheOREM 3.1. Let $H$ be an arbitrary Hilbert Space, $T$ a linear operator on $H$ with adjoint $T^{*}$. Then

1. If $\lambda$ is in the residual spectrum of $T, \bar{\lambda}$ is in the point spectrum of $T^{*}$.

2. If $\lambda$ is in the point spectrum of $T, \bar{\lambda}$ is in the point or residual spectrum of $T^{*}$. 
3. If $\lambda$ is in the continuous spectrum of $T, \bar{\lambda}$ is in the continuous spectrum of $T^{*}$.

4. If $\lambda$ is in the resolvent set of $T, \bar{\lambda}$ is in the resolvent set of $T^{*}$.

For a proof of 1 or 2 see Stone [2, p. 143].

If $\lambda$ is in the resolvent set of $T$, then $[T-\lambda]^{-1}$ exists as a bounded operator. Thus $\left([T-\lambda]^{-1}\right) *$ exists and is bounded $[2$, Theorem 2.29 , p. 64]. Since ${ }^{*}$ and ${ }^{-1}$ can be interchanged [2, Theorem 2.7, p. 43], $\left[T^{*}-\bar{\lambda}\right]^{-1}$ exists and is bounded and $\bar{\lambda}$ is in the resolvent set of $T^{*}$.

If $\lambda$ is in the continuous spectrum of $T$ and $\bar{\lambda}$ is in the point spectrum of $T^{*}$, then 2 gives a contradiction. If $\bar{\lambda}$ is in the residual spectrum of $T^{*}$, then 1 gives a contradiction. If $\bar{\lambda}$ is in the resolvent set of $T^{*}$, then 4 gives a contradiction. Thus $\bar{\lambda}$ is in the continuous spectrum of $T^{*}$.

It is easy to verify that if $\lambda$ is not on the positive semi-axis $\lambda \geqq 0$ and $N(s)=0$, that $\bar{u}(x, s)$ is an eigenfunction of $L^{*}$ with eigenvalue $\bar{\lambda}$. Thus Theorem 3.1 leads us to the following

THEOREM 3.2. The spectrum of $L^{*}$ consists of

1. Eigenvalues $\bar{\lambda}$ with eigenfunctions $\bar{u}(x, s)$ whenever $N(s)=0$ and $\lambda=s^{2}$ is not on the positive semi-axis $\lambda \geqq 0$.

2. Continuous spectrum on the positive semi-axis $\lambda \geqq 0$ unless path $N(s)$ and $N(-s)$ vanish together in which case $\lambda$ is possibly in the point spectrum.

3. The residual spectrum of $L^{*}$ is empty.

REMARK. It is evident that the Green's function for $L^{*}$ is $\bar{V}(\bar{\lambda}, \xi, x)$ and that the nonhomogeneous expansion derived from $V$ in [1] is the $L^{*}$ eigenfunction expansion.

\section{REFERENCES}

1. Allan M. Krall, $A$ nonhomogeneous eigenfunction expansion, Trans. Amer. Math. Soc. 117 (1965), 352-361.

2. M. H. Stone, Linear transformations in Hilbert space, Amer. Math. Soc. Colloq. Publ. Vol. 15, Amer. Math. Soc., Providence, R. I., 1932.

Pennsylvania State University 